Recepción: 01 / 03/ 2018

Aceptación: 15 / 05 / 2018

Publicación: 07 / 08 / 2018

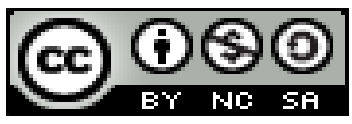

Ciencias de la Salud

Artículo Científico

\title{
Consumo alimentario y estado nutricional de los adultos mayores atendidos en el hospital básico Jipijapa en el área de medicina interna periodo noviembre 2017 abril 2018
}

Food consumption and nutritional status of the elderly served in the basic hospital Jipijapa in the area of internal medicine period November 2017 April 2018

Consumo alimentar e estado nutricional de idosos atendidos no hospital básico de Jipijapa na área de medicina interna novembro 2017 abril 2018

\author{
Yasmin A. Castillo-Merino I \\ yasminac@gmail.com \\ Monserrate Merino-Conforme ${ }^{\text {II }}$ \\ monserratemc@gmail.com \\ Deysi Delgado-Lopez III \\ deysi.delgado@gmail.com
}

\author{
Manuel M. Chiquito-Pisco IV \\ manuelm@gmail.com \\ Leonela L. Ponce-Lino v \\ leonelal.ponce@gmail.com \\ Bolívar R. Hidalgo-Alvarez VI \\ bolívar.hr@gmail.com
}

Correspondencia: yasmina@gmail.com

I. Lic. En Enfermería. Mg en Gerencia en Administración en Salud; Carrera de Enfermería, Facultad Ciencias de la Salud, Universidad Estatal del Sur de Manabí, Jipijapa, Ecuador.

II. Lic. En Enfermería. Mg en Gerencia en Salud para el Desarrollo Local; Carrera de Enfermería, Facultad Ciencias de la Salud, Universidad Estatal del Sur de Manabí, Jipijapa, Ecuador.

III. Lic. En Enfermería. Mg en Gerencia en Salud para el Desarrollo Local; Carrera de Enfermería, Facultad Ciencias de la Salud, Universidad Estatal del Sur de Manabí, Jipijapa, Ecuador

IV. Médico Familiar Distrito de Salud, Centro de Salud Jipijapa. Docente de Post Grado Medicina Pontificia Universidad Católica del Ecuador Quito, Ecuador.

V. Lic. En Enfermería. IESS, Jipijapa, Ecuador.

VI. Lic. En Enfermería. IESS, Jipijapa, Ecuador. 
Consumo alimentario y estado nutricional de los adultos mayores atendidos en el hospital básico Jipijapa en el área de medicina interna periodo noviembre 2017 abril 2018.

\section{Resumen}

Los requerimientos energéticos declinan con la edad en asociación con la pérdida de masa magra y la disminución de la actividad física. La ingesta energética disminuye más aún en los más adultos mayores a causa de incapacidades que limitan su actividad física. Los numerosos cambios físicos, psíquicos y sociales que acompañan al envejecimiento y la mayor prevalencia de enfermedades crónicas, también contribuyen a esta situación que conlleva al incremento de patologías que afectan a la población de la tercera edad. En este sentido el trabajo de investigación tiene por objetivo determinar el consumo alimentario y estado nutricional de los adultos mayores atendidos en el Hospital Básico Jipijapa. El diseño metodológico en la cual se incluye el estudio inducción, metodológico y de campo ya que se realizó la investigación de noviembre 2017 a abril 2018; el universo de estudio corresponde a 87 personas adulto mayores. Se encuentra la presentación de datos de la investigación donde se da a conocer el análisis de los resultados de las encuestas realizadas a los adultos mayores, una vez recopilada la información fue tabulada y procesada donde se detectó que el requerimiento nutricional de la población en estudio no se lleva correctamente en cuanto al consumo de proteínas y minerales, así también, las principales patología son las crónicodegenerativas. Es por esto que llego a diseñar la propuesta que es diseñar una guía alimentaria con los requisitos nutricionales de los adultos mayores para mantener una adecuada calidad de vida.

Palabras claves: Adulto mayor, evaluación de requerimiento nutricional, consumo alimentario y estado nutricional. 


\begin{abstract}
The energy requirements decline with age in association with the loss of lean mass and the decrease in physical activity. The energy intake decreases even more in older adults due to disabilities that limit their physical activity. The numerous physical, psychological and social changes that accompany aging and the higher prevalence of chronic diseases also contribute to this situation that leads to an increase in pathologies that affect the elderly population. In this sense, the research work aims to determine the food consumption and nutritional status of the elderly served in the Jipijapa Basic Hospital. The methodological design in which the induction, methodological and field study is included since the research was conducted from November 2017 to April 2018; the study universe corresponds to 87 elderly people. It is the presentation of data from the research where the analysis of the results of the surveys made to the elderly is presented, once the information was collected it was tabulated and processed where it was detected that the nutritional requirement of the population under study was not It is properly carried out in terms of the consumption of proteins and minerals, and also, the main pathologies are chronic degenerative. That is why I came to design the proposal that is to design a food guide with the nutritional requirements of the elderly to maintain an adequate quality of life.
\end{abstract}

Key words: Bigger adult, evaluation of nutritional requirement, I consummate alimentary and nutritional state. 
Consumo alimentario y estado nutricional de los adultos mayores atendidos en el hospital básico Jipijapa en el área de medicina interna periodo noviembre 2017 abril 2018.

\section{Introducción.}

El consumo alimentario juega un papel muy importante en el proceso de envejecimiento a través de la modulación de cambios asociados al envejecimiento en diferentes órganos y funciones del organismo; de allí la importancia de evaluar el estado nutricional en el adulto mayor para determinar debido a que factores se presentan deficiencias nutricionales. El estado de alimentación está determinado por los requerimientos y la ingesta; ellos a su vez son influenciados por otros factores como la actividad física, los estilos de vida, la existencia de redes sociales y familiares, la actividad mental y psicológica, el estado de salud o enfermedad y restricciones socioeconómicas ${ }^{1}$.

Según Fondo de Población de las Naciones Unidas (FNUAP)², el aumento de la población de adultos mayores a nivel mundial es objeto de discusión en este momento. A partir de 1950 la proporción de adultos mayores de 65 años en los países desarrollados ha aumentado desde un $8 \%$ hasta un $14 \%$ en la actualidad y se estima que éstos llegarán a conformar el $25 \%$ en el año 2050.

Se estima que en el último censo poblacional efectuado por el Instituto Nacional de Estadística y Censo (INEC), en el Ecuador existen 1'229.089 adultos mayores (personas de más de 60 años), la mayoría reside en la sierra del país (596.429), seguido de la costa (589.431); en su mayoría son mujeres $(53,4 \%)$ y la mayor cantidad está en el rango entre 60 y 65 años de edad ${ }^{3}$.

Este grupo etario es muy heterogéneo y los cambios son individuales, no existe un momento biológico a partir del cual se inicie la tercera edad, reconociéndose el papel de la herencia genética, aunque no menos importantes son otros aspectos como una adecuada alimentación, las enfermedades asociadas, el ambiente y los hábitos de vida saludables. Los requerimientos energéticos declinan con la edad en asociación con la pérdida de masa magra y la disminución de la 
actividad física. La ingesta energética disminuye más aún en los más adultos mayores a causa de incapacidades que limitan su actividad física.

Dentro de las necesidades que deben asegurarse en el anciano, tienen una importancia especial el agua y las vitaminas. El agua es uno de los nutrientes más importantes requeridos para la mantención de la homeostasis en ancianos, debido a su papel esencial en la regulación del volumen celular, el transporte de nutrientes, la remoción de desechos y la regulación de la temperatura. La disminución del agua corporal que ocurre con el envejecimiento (80\% al nacimiento y 60 a $70 \%$ en ancianos), los hace especialmente lábiles a la pérdida de agua, lo cual tiene profundas consecuencias sobre la salud de este grupo ${ }^{4}$.

Los adultos mayores son el grupo de población de mayor crecimiento en todo el mundo, pero esto no significa necesariamente que su calidad de vida sea mejor. Así como ha aumentado el número de ancianos, aparentemente también ha aumentado entre ellos la incidencia de problemas relacionados con estado alimentario, como la desnutrición, el sobrepeso y la obesidad ${ }^{5}$.

La alimentación juega un papel protagónico en esa situación, ya que está relacionada con la aparición y mantenimiento de enfermedades, así también, el hecho de vivir más años no significa tener un mejor estado de alimentación, puesto que una gran proporción del adulto mayor tiene problemas de desnutrición, sobrepeso u obesidad, con repercusiones fatales en su calidad de vida y en los costos médicos de atención. Además de esto, los sistemas de salud en todo el mundo están en crisis y difícilmente podrán sostener el reto que implica una población anciana con problemas crónicos, discapacidades y además, con problemas de nutrición que afectan aún más su vida y los costos de los sistemas de salud ${ }^{6}$. 
Consumo alimentario y estado nutricional de los adultos mayores atendidos en el hospital básico Jipijapa en el área de medicina interna periodo noviembre 2017 abril 2018.

A partir de los 60 años las necesidades calóricas (calorías a ingerir por día) disminuyen un $10 \%$ por cada década de la vida, consecuencia de que tanto el gasto energético basal (en reposo) como la actividad física disminuyen con la edad. Pero, además, con la edad hay cambios en las necesidades de algunos nutrientes. Existe aumento de las necesidades proteicas (de 1 a 1,2 g/kg/día) y parecen estar incrementados los requerimientos de vitamina B1, vitamina B12, vitamina C, vitamina D y calcio. Pues la crisis de los sistemas de salud al envejecimiento de la población la atención mundial se enfoca a lograr construir o identificar sistemas de salud costo- eficientes que permitan prevenir al anciano y promuevan su dignidad, su independencia y su calidad de vida. Con este fin, se pretende diseñar programas de alimentación dirigidos al adulto mayor, ya que dentro de los principales factores de riesgo modificables para la prevención de las enfermedades crónicas y sus complicaciones, está la nutrición ${ }^{7}$.

La desnutrición no es una consecuencia inevitable del envejecimiento, pero con el aumento de la edad se producen algunos cambios en la composición del organismo. En las personas mayores sanas disminuye la masa muscular magra aquella formada por (huesos y músculos), fundamentalmente aumenta el contenido de grasa a casi el doble que en la edad adulta mayor ${ }^{8}$.

Es cierto que las personas mayores son el segmento de la población que tiene una mayor preocupación por unos hábitos alimentarios saludables y a elegir aquel tipo de alimentos que mejor contribuyan al incremento de su salud y aumento de la longevidad. Pero conviene subrayar que todas estas investigaciones y propuestas se aplican a todas las edades y se recomienda seguirlas en todas las etapas de la vida y no solamente en la vejez ${ }^{9}$. 


\section{Materiales y métodos.}

La investigación fue de tipo descriptivo - analítico, el objetivo principal de la investigación consiste en determinar el consumo alimentario y estado nutricional de los adultos mayores atendidos en el Hospital Básico Jipijapa en el área de medicina interna durante el período noviembre 2017 abril de 2018, como variable independiente tenemos el consumo alimentario mientras que la variable dependiente adulto mayor y estado nutricional, los indicadores a medir tenemos: medidas antropométricas, peso en relación a la talla, talla en relación en la edad, edad, IMC, alimentación, factores de riesgo.

La población la constituyen 111 pacientes ingresados en el Hospital Básico de Jipijapa en el Área de Medicina Interna, Periodo Noviembre 2017 - Abril 2018, la muestra es de 87 pacientes determinado mediante el muestreo investigativo. Los criterio de inclusión fue la población seleccionados mediante el muestro, se excluirán a pacientes adultos mayores hospitalizados que les dificultara colaborar y no desearon participar en la investigación. Las técnicas que se llevaron a cabo mediante esta investigación fueron encuesta dirigidas a los adultos mayores que ingresaron al Hospital Básico de Jipijapa al área de medicina interna y técnicas de observación mediante la revisión de los expedientes. La información recopilada se procesó en MS Excel 2010 y los resultados se expresaron en forma de tablas.

Los procesos metodológicos de la investigación son de inducción que se utilizó para describir los casos de dichas patologías situadas en el Hospital Básico de Jipijapa, método bibliográfico porque permitió conocer, comparar, ampliar y profundizar los diferentes enfoques teóricos y conceptualizaciones en la fase de recopilación de información sobre temas relacionados de consumo alimentario y estado nutricional de los adultos, basados en libros de protocolos del 
Consumo alimentario y estado nutricional de los adultos mayores atendidos en el hospital básico Jipijapa en el área de medicina interna periodo noviembre 2017 abril 2018.

adulto mayor, revistas de carácter científico e internet. Investigación de campo se utilizó para la exploración de nuevos conocimientos en el campo de la realidad social, bien a estudiar una situación para identificar necesidades y problemas de los adultos mayores atendidos en el Hospital Básico de Jipijapa en el área de medicina interna.

\section{Resultados.}

De acuerdo a las técnicas utilizadas se obtuvo que el $61 \%$ de los adultos mayores pertenecieron al sexo femenino y con el 39\% masculino, en lo que corresponde a la edad el $44 \%$ indica que existe un rango entre 65 a 75 años de edad, seguido del 34\% en el rango de 76 a 86 años de edad y de $87 \mathrm{y}+$ años de edad con un $22 \%$

En la valoración del índice de masa corporal se verifico que el 72\% de los adultos mayores poseen peso Normal, el 12\% un Sobrepeso, 8\% Obesidad Mórbida, 6\% Obesidad, y un 2\% en bajo peso para la edad.

A los usuarios adultos mayores hospitalizado en el área de medicina interna se pudo constatar que la mayoría ingieren alimentos 3 veces al día con un $54 \%$, y un $22 \%$ ingieren alimentos más de 5 veces al día, el 18\% ingieren alimentos 4 veces al día, un $4 \%$ ingieren alimentos dos veces al día, y el 2\% responden ingerir una vez al día.

Con respecto a la pregunta sobre el tipo de alimentación diaria se obtuvo que 23 pacientes consumen carbohidratos con un porcentaje de 26\%, 20 pacientes respondieron consumir grasas $23 \%, 19$ consumen vitaminas con el 22\%, 15 pacientes consumen minerales como leche, pescado y mariscos con un 17\%, y se concluye con la respuesta de la encuesta que 10 adultos mayores prefieren consumir proteínas como nueces y huevos, con un porcentaje de $12 \%$. 
Mediante la recolección de datos y la observación directa hacia los usuarios adultos mayores se obtuvo una frecuencia de 69 pacientes que comen solos sin dificultad que corresponde al 79\%, mientras 10 adultos mayores ingresados respondieron tener dificultad que representa el 12\%, y 8 pacientes adultos mayores necesitan ayuda de su familiar en un 9\%. En su gran mayoría los adultos mayores y de acuerdo a las encuestas realizadas a 87 pacientes respondieron vivir con su familiar y en un menor porcentaje que no vivan con familiares.

Se determinó el nivel de instrucción de los adultos mayores donde se pudo observar que la gran mayoría de los adultos mayores tienen un nivel primario con 31 pacientes que representa el 36 $\%, 26$ respondieron ser analfabetos con el 30\%, mientras que 23 tienen un nivel secundario que corresponde al $26 \%$, y 7 pacientes respondieron tener un nivel superior con un $8 \%$.

En cuanto al índice de patologías se evidencia que el $41 \%$ los adultos mayores sufren de Hipertensión arterial, un 36\% de Diabetes mellitus, el 13\% en Osteoporosis y el $10 \%$ en Neumonía, así también se pudo apreciar que el $46 \%$ de los adultos mayor toman medicamentos por prescripción médica, el $33 \%$ lo realizan con criterio y conveniencia y el $21 \%$ con sugerencias de amigos y vecinos.

De acuerdo a la actividad física realizada por los adultos mayores se obtuvo que el $56 \%$ no practican actividad física en sus tiempos libres, mientras que el $44 \%$ si lo practican. En base a las actividades realizadas de su vida diaria el $40 \%$ de los adultos mayores respondieron que su actividad diaria es cantar y bailar, el $26 \%$ se pasan en comunicación con sus familiares por medio del teléfono, el 18\% realiza su actividad en casa subiendo las escaleras, y el $16 \%$ refiere que salen a trotar. 
Consumo alimentario y estado nutricional de los adultos mayores atendidos en el hospital básico Jipijapa en el área de medicina interna periodo noviembre 2017 abril 2018.

De los pacientes encuestados 52 respondieron tomar líquido vital de 2 a 4 vasos de agua diario con un porcentaje de $60 \%, 23$ respondieron que ingieren de 5 a 7 vasos de líquido vital diariamente que corresponde al 26\%, y $14 \%$ que representa 12 pacientes responden que si toman de 8 a 10 vasos de agua diariamente bajo prescripción médica .

\section{Discusión.}

El incremento de la esperanza de vida junto con el descenso de la natalidad, implica un envejecimiento de la población y con ello nuevos retos de intervención dirigidos a este grupo denominados adultos mayores. De aquí la importancia de desarrollar estudios que nos permitan contar con información sobre este grupo.

Una vez analizados los resultados de la investigación de campo considerando la teoría de los autores se llegan a precisar que de acuerdo a los datos obtenidos de la población de los adultos mayores permiten verificar que la mayoría se encuentra en las edades de 65 a 87 y más años, el consumo alimenticio proyecta a un peso normal, donde el sexo femenino se encuentra en un porcentaje $61 \%$.

Los hábitos alimentarios influyen en el estado nutricional del adulto mayor, debido a que gran parte de la población encuestada posee hábitos alimentarios saludables e ingesta adecuadas de macro y micronutrientes, lo cual se refleja en el alto porcentaje de adultos mayores con peso normal. Cabe destacar que el índice de masa corporal es solo uno de los parámetros para evaluar el estado nutricional del individuo.

Se identificó que las patologías más relevantes que padecen en los adultos mayores son: Hipertensión Arterial, Diabetes, Accidente Cerebro Vascular, Artritis, osteoporosis y neumonía, que 
se puede atribuir a una actividad sedentaria y hábitos nocivos; y a esto se da que las enfermedades prevalentes son las Hipertensión y Diabetes que se aprecian en las encuestas realizadas.

Teniendo en cuenta los resultados del análisis concordancia entre paciente y familiar se puede observar que la mayoría de las variables presentan resultados satisfactorios, lo cual indica que las respuestas del adulto mayor en el cuestionario tienen valor de veracidad, por tanto se tuvieron en cuenta para los resultados de esta investigación.

Ejecutadas las operaciones estadísticas se llega a la conclusión que la hipótesis está en el nivel de aceptación. La comprobación de la hipótesis se demuestra y se llega a la conclusión que se diseña una guía alimenticia con los requerimientos nutricionales de los adultos mayores para mantener una adecuada calidad de vida. 


\section{Bibliografía.}

1. Banco Internacional de Desarrollo. Informe Final: Modelo de cuidado de personas adultas dependietes cuidadoras y su participación en el mercado laboral; 2014.

2. Servicio Nacional del Consumidor-SERNAC. Nutrición y cuidados del adulto mayo. Recomendaciones para una alimentación saludable. Servicio Nacional del ConsumidorSERNAC. Chile; 2004. Recuperado de https://fiapam.org/wpcontent/uploads/2012/10/Nutricion-y-Cuidados-del-Adulto-Mayor.pdf

3. Instituto Nacional de Estadística y Censo-INEC. Encuesta de Salud, Bienestar del adulto mayor [Online]. Recuperado de: http://www.ecuadorencifras.gob.ec/encuesta-de-saludbienestar-del-adulto-mayor/

4. Ayres Carvalho, J. Conceptos y prácticas en salud pública: algunas reflexiones. Rev. Fac. Nac. Salud Pública 2002;20(2): 67-82. Recuperado de http://www.redalyc.org/pdf/120/12020206.pdf

5. Bernal - Orozco M., Vizmanos B., Celis J. La nutrición del anciano como un problema de salud pública. Antropo. 2008; 15: 43-55. Recuperado de http://www.didac.ehu.es/antropo/16/16-6/BernalOrozco.pdf

6. De la Cruz, E. La Alimentación: un acto por repensar, reflexionar y redefinir desde la perspectiva educativa. [Online]. Fundación Bengoa. Recuperado de: https://www.fundacionbengoa.org/informacion_nutricion/alimentacion.asp

7. Azcona ÁC. Nutrición y Dietética: manual. Madrid: Universidad Complutense de Madrid, Departamento de Nutrición; 2012.

8. Docsalud. Consecuencias de la malnutrición en el adulto mayor. [Online]. 2011

9. José Miguel Guzmán sh. Redes de apoyo social de personas mayores: marco teórico conceptual. Ponencia. México: Universidad Nacional Autónoma de México, 2013. 